\title{
$\begin{array}{ll}\text { Research Square } & \begin{array}{l}\text { Preprints are preliminary reports that have not undergone peer review. } \\ \text { They should not be considered conclusive, used to inform clinical practice, } \\ \text { or referenced by the media as validated information. }\end{array}\end{array}$
}

\section{Food Security and Its Association with Socioeconomic Status and Diet Diversity in Free Living Elderly in Tehran, Iran}

Fatemeh Pourebrahim

Shahid Beheshti University of Medical Sciences School of Nutrition and Food Technology

Nasrin Omidvar ( $\square$ omidvar.nasrin@gmail.com)

Shahid Beheshti University of Medical Sciences School of Nutrition and Food Technology https://orcid.org/0000-0001-7305-0461

Arezoo Rezazadeh

Shahid Beheshti University of Medical Sciences School of Nutrition and Food Technology

Hassan Eini-Zinab

Shahid Beheshti University of Medical Sciences School of Nutrition and Food Technology

Pedram Shirani

Shahid Beheshti University of Medical Sciences School of Nutrition and Food Technology

\section{Research article}

Keywords: Food Security, Elderly, USHFSSM, Dietary Diversity Score, Socioeconomic factors, Iran

Posted Date: May 20th, 2020

DOI: https://doi.org/10.21203/rs.3.rs-28947/v1

License: (1) This work is licensed under a Creative Commons Attribution 4.0 International License. Read Full License 


\section{Abstract \\ Backgrounds:}

Food security is one of the most important factors affecting food access and diet diversity. Older age is a period when adequate and diverse dietary intake is a challenge and there is a high risk for malnutrition. This study aimed to investigate the association between food security, dietary diversity and socioeconomic factors among the free-living elderly in the city of Tehran.

\section{Methods}

In this cross-sectional study, 583 elderlies (279 men and 304 women) aged 60-80 years were selected through clustered systematic multistage sampling method from 10 districts of Tehran city. Food security status of the subjects was determined using locally validated version of the United States Household Food Security Survey Module )US-HFSSM( questionnaire. Socio-economic status was assessed by a questionnaire. Dietary intake of the subjects was evaluated using two 24-hour recalls (one weekday and one week-end) through face-to-face interviews. Dietary Diversity Score (DDS) was calculated using the FAO 2010 guideline. Multinomial logistic regression was applied for the analysis.

\section{Results}

The average age of participants was $67.87 \pm 5.86$ years. Based on US-HFSSM, $56.9 \%$ of the elderly were food secure; while $25.7 \%, 14.2 \%$ and $3.2 \%$ suffered from food insecurity $(\mathrm{FI})$ without hunger, with moderate hunger, and with severe hunger, respectively. There was no association between $\mathrm{FI}$ and DDS, even after controlling for confounders. Multinomial logistic regression models suggested that after adjusting for age and gender, being illiterate increased the possibility of different levels of food insecurity [(OR=3.107, 95\% Cl $=1.672-5.775),(\mathrm{OR}=2.736,95 \% \mathrm{Cl}=$ $1.261-5.935)$ and $(\mathrm{OR}=6.193,95 \% \mathrm{Cl}=1.368-28.039)]$, respectively. Also, $\mathrm{Fl}$ with mild hunger was associated with total household income $(\mathrm{OR}=2.744,95 \% \mathrm{Cl}=1.100-6.846)$, while $\mathrm{FI}$ with severe hunger was significantly associated with Fars ethnicity $(\mathrm{OR}=0.146,95 \% \mathrm{Cl}=0.051-$ $0.424)$.

\section{Conclusions}

Overall, socio-economic status and demographic characteristics were the predictors of Fl among the elderly, while DDS was not associated with FI.

\section{Introduction}

In recent decades, increasing life expectancy and decreasing fertility and birth rates have increased the proportion of elderly in the general population and this trend will continue over the next decades [1]. The World Health Organization estimates that by 2050, the elderly population or those crossing the age of sixty will reach 1.5 billion [4]. In Iran, as a country experiencing population transitions due to health and education developments, the population of elderly is growing significantly. While the percentage of the elderly population in 2011 was $8.3 \%$, it is expected to reach $10.3 \%$ in 2025 [3]. With the growing population of elderly, the issue of health and well-being in the society is finding new and wider dimensions every day [2]. For all those who have survive life events and go through youth and middle age, this time will come true. Therefore, ensuring health and well-being in this age group and improving their quality of life has become a priority from public health, as well as economic planning point of view.

Food security is one of the most important social, economic and political issues in different countries of the world, especially in middle and low income countries [6]. Food Security, as defined by the Food and Agriculture Organization (FAO), is the situation that all people have access to adequate, healthy and nutritious food at all times in order to meet their nutritional needs and preferences for an active and healthy life [7]. Food insecurity and hunger are of particular interest to researchers and policymakers because of the direct and indirect costs that they bring to individuals and therefore to society [9]. Among the elderly, in addition to old age, living alone, being disabled or physically incapable of doing things, especially buying and preparing food, and suffering from certain diseases, make them more vulnerable to food insecurity than younger individuals [8].

Food security affects food choices and food intake [9-10], as well as diet diversity [11]. Diet diversity refers to the number of food items consumed over a period of time [12]. A diverse diet is associated with a higher intake of micronutrients [13]. Socioeconomic and demographic factors, including household size, house ownership, educational levels, access to health and insurance services, and total household income are the most important factors affecting food security [14]. Studies have shown that there is a positive relationship between dietary diversity 
and access to energy and food at both individual and household levels [16]. The results suggest that increasing income in large cities is a factor in increasing individual diet diversity [17].

Several studies have examined the relationship between food security and food diversity and also between socioeconomic factors and food security among the elderly $[19,24-25]$. They found that low income and low educational level were the most important predictors of elderly FI in the world [24-25]. A study examining the relationship between food security and dietary diversity in people over the age of 40 in a district in Tehran city found that Dietary Diversity Score (DDS), as one of the indicators of healthy eating was associated with food security status; and as the level of food security increased, DDS, especially fruits and vegetables DDS increased [19]. In another study in Shiraz, an inverse relationship between food insecurity and consumption of meat, milk, fruits, and vegetables scores was reported [20]. Finally, a study on Food insecurity and some associated risk factors in Tehranian elderly women showed that food insecurity was more frequent among women who were single, had swallowing problem and those with low to moderate socioeconomic status [18].

Nutrition and health status of the elderly are different from other age groups in the society, due to their higher vulnerability to diseases and adverse health conditions [15]. Given the prevalence of food insecurity and its risk factors in the elderly and its impact on food intake and dietary diversity, it is important to identify major determinants of food security in the elderly and its association with diet quality. Few studies have examined food security status of the free-living elderly in Iran and specifically in urban settings. According to the latest census, Tehran, the capital and the largest city of the country, has a population of 12,183,391 people, of whom 1,200,123 are over 60 years of age [5]. The present study aimed to investigate the relationship between food security and socioeconomic factors and dietary diversity among the elderly living in Tehran.

\section{Materials And Methods \\ Participants}

This study was part of a large project entitled "Situation analysis of lifestyle of free-living elderly residents of Tehran". In this cross-sectional study, 583 (304 Women, 279 men) free living elderly residing in Tehran city were selected in 2017-2018 from those who were willing to participate in the study, were not residence in nursing homes or any institution, had Iranian nationality, aged between 60 and 80 years, with no severe illness or metabolic diseases such as cancer, end-stage kidney disease and severe metabolic and cognitive disorders, and were able to speak and communicate.

The number of subjects required was calculated with regard to food security status and dietary diversity of the elderly as the main variables, using Qomi et al. study in district 13 of Tehran [19]. Using this information and by adding Power of $90 \%$, the calculated sample size was 446, and with counting $10 \%$ of the sample drop out, it was increased to 490 ; finally, to increase the accuracy of the study the sample size of 583 was considered.

The participants were selected by clustered systematic multi-stage sampling from public health centers (PHCs) [60\% of the sample size]), community centers (called Saraye-Mahalleh) [20\% of the sample] and mosques communities [20\% of the sample] according to the demographic weight of the selected districts. Tehran districts were categorized into four zones based on socio-economic factors [28]. Based on this ranking, districts 1, 3, 6 and 7 were ranked as more developed zone, districts 2, 4, 5, 8, 11, 12, 13, and 14 as medium-upward developed zone, districts 9, 15, 19, 20, 21 as medium-downward developed and districts 10, 16, 17, 18 and 22 as least developed zones [28].

\section{Measurements}

\section{Demographic and socioeconomic characteristics}

From each elderly, a household socio-economic questionnaire was completed through face to face interview [46]. The questionnaire consisted of socio-demographic information, including age, gender, ethnicity (Fars, Azeri and others), educational level (illiterate and elementary, middle and high school, High school diploma and higher), marital status (single, married, widowed), relationship to the head of the household (head, spouse and other), household size, number of children, as well as questions on economic status, including employment status (unemployed, employed, housewife, retired and other), residence district, home ownership status (owner, other), house size, number of rooms, living facilities (vehicle, television, freezer, refrigerator, gas,...), and receiving food aids or financial support from organizations and charities. Also, total household income was obtained indirectly.

\section{Food Security}

Food security of the studied elderly was assessed using US-HFSSM questionnaire validated for Iranian elderly by Rafiei et al. [22]. The USHFSSM questionnaire includes 10 items that measure the levels of insecurity and occurrence and non-occurrence and the frequency of recurrence of the problem in the last 12 months [23]. The first question contained three response options, including often (occurrence per

Page $3 / 16$ 
month), sometimes (occurrence in some months) and never/rarely (non/one or two months of the year). In two questions (number 4B and 8B), frequency of occurrence were asked in the form of almost every month, some months or up to two months in the past 12 months; the rest of the questions were yes/no questions. These questions were related to three different areas of access to food, including worrying and uncertainties about food stocks, inappropriate quality, low diversity and mismatch with food preferences and getting insufficient food [23].

Dietary intake

Dietary intake of the subjects was assessed using two non-consecutive 24-hour recalls (one week day and one weekend) by multiple-pass method [26] through interview by trained nutritionists using a questionnaire. First, elderlies were asked to remember their food and drink intake during the past 24 hours after waking up (generally a quick list). Then, they were asked about the details of what they had eaten for instance for breakfast cereals, milk, etc. In the next round, they were asked about the details of what they had eaten, e.g. percentage of milk fat or question about brand, package weight purchased, etc. and how much of each food they had consumed, time of consumption, preparation method(s) and how and where it was consumed. Finally, the questionnaire was browsed along with the interviewee for forgotten or misreported items, and also queries to estimate the amount of foods consumed, using home scales, geometric shapes, rulers, food models, and a food album [27]. If all the answers were not ready, they were asked by phone on the next day [26].

\section{Dietary Diversity Score}

Using the data from the 24-hour recalls, the FAO (2013) Dietary Diversity Score (DDS) was calculated [9]. Based on this approach, all foods were divided into 9 food groups: 1) cereals, 2) milk and dairy products, 3) vitamin A-rich fruits and vegetables, 4) green leafy vegetables, 5) other fruits and Vegetables, 6) Meat, fish and seafood, 7) Organ meats, 8) Eggs, and 9) Nuts, seeds and beans. DDS was calculated by consuming at least $15 \mathrm{~g}$ of one food item for each of the listed food groups during the two reported days. DDS was the total score of the food groups and ranged from 0 to 9 which was classified into two levels: low ( $\leq 3)$ and high (>3) [40].

\section{Statistical Analysis}

Data were analyzed by SPSS version 21. After data cleaning, the normality test was used to determine if the distribution of variables is normal. The number of rooms and house size were divided by household dimension and their per capita value was calculated separately. Also, each home living facility was scored 1 to 10 , based on its financial weight. The total score was calculated as "Facility Score". Finally, the sum of per capita number of rooms, per capita house size and facility score were defined as "welfare index".

For calculating DDS, those with incomplete 24-hour recall questionnaires and/or over or under-reporting of energy (below 500 and above $3500 \mathrm{kcal}$ in women, below 800 and over $4000 \mathrm{kcal}$ in men) [21] were excluded from the study.

Dietary intake, food security status, demographic characteristics and SES were analyzed by descriptive statistics. To test the association between independent and dependent variables, multinomial and binary logistic regression tests were used.

\section{Results}

Demographic characteristics of the study participants are presented in Table 1. Overall, 583 elderly participated in this study with mean age of $67.87 \pm 5.86$ years of whom $304(52.1 \%)$ were women. More than half of the participants belonged to Fars ethnic group (56.5\%). The percentage of women who lived alone or were single were almost $3(17.8 \%$ vs. $5.0 \%)$ and $7(34.2 \%$ vs. $5.4 \%)$ times more than their male counterparts, respectively. On the other hand, the percentage of men heading a household were 2.5 times more than women. The average household size was $3.01 \pm 1.41$, and total number of children and number of children living with the elderly were, $3.83 \pm 1.75$ and $0.94 \pm 1.05$, respectively. 
Table 1

Demographic characteristics of the elderly by sex, Tehran, Iran

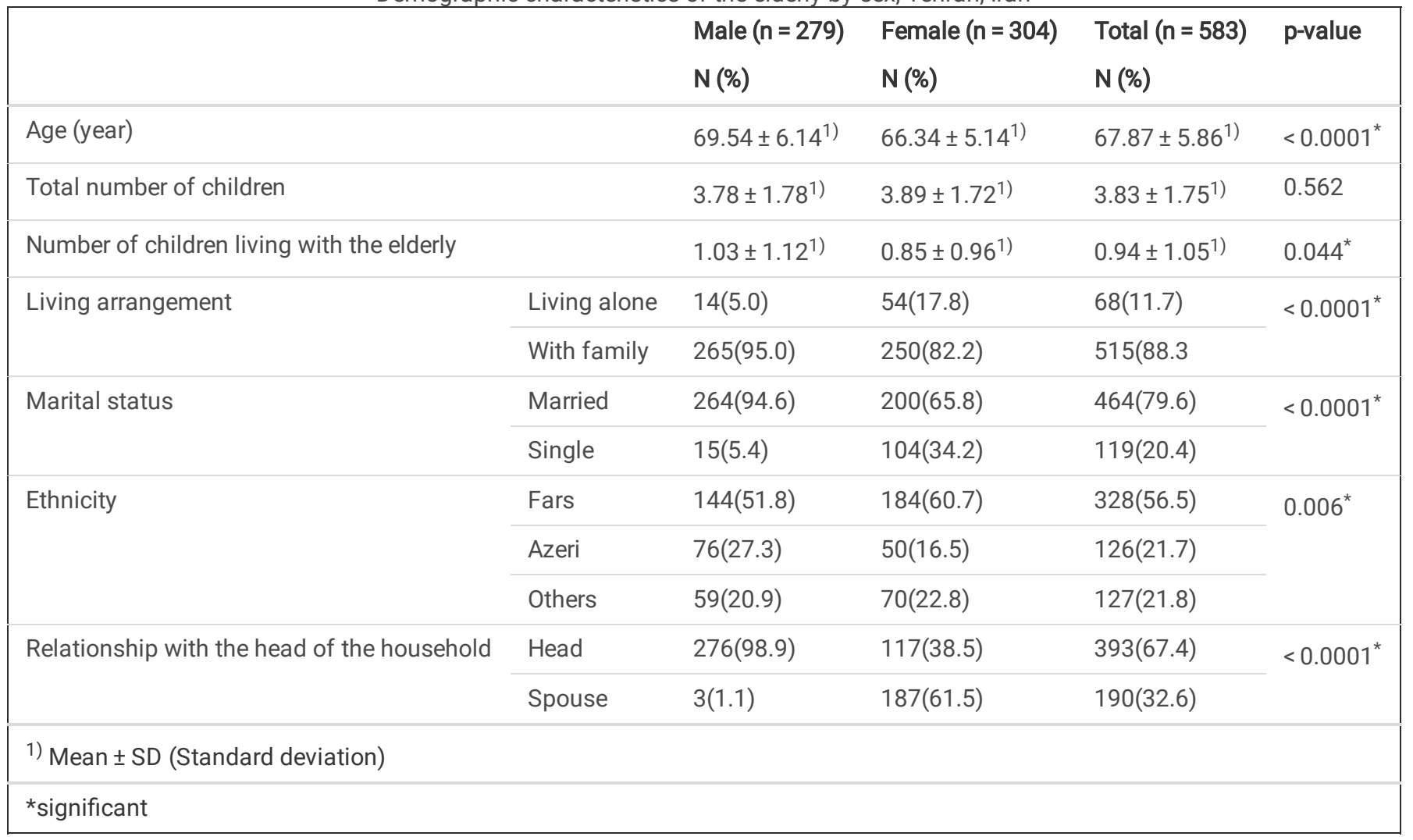

Socio-economic characteristics of the participants are shown in Table 2. More than half of the participants were illiterate or with elementary education (52.1\%); about half were retired (44.9\%) of whom most were men (74.6\%). Most of the participants owned the house they lived in (84.3\%). Household income in about half (46.7\%) of the participants was between 74 to 148.15 US\$ per month. The percentage of women with monthly household income less than 74 US\$ was almost twice (23.2\% vs. $10.0 \%)$ elderly men; while the percentage of men with monthly household income of 148.15 to 222.22 US\$ were about twice women. More than half of the participants lived in the medium developed zone (58.3\%). The mean welfare index and household size were $63.86 \pm 30.14$ and $3.01 \pm 1.41$, respectively, with no sex difference. 
Table 2

Socio-economic characteristics by sex of the participants, Tehran, Iran

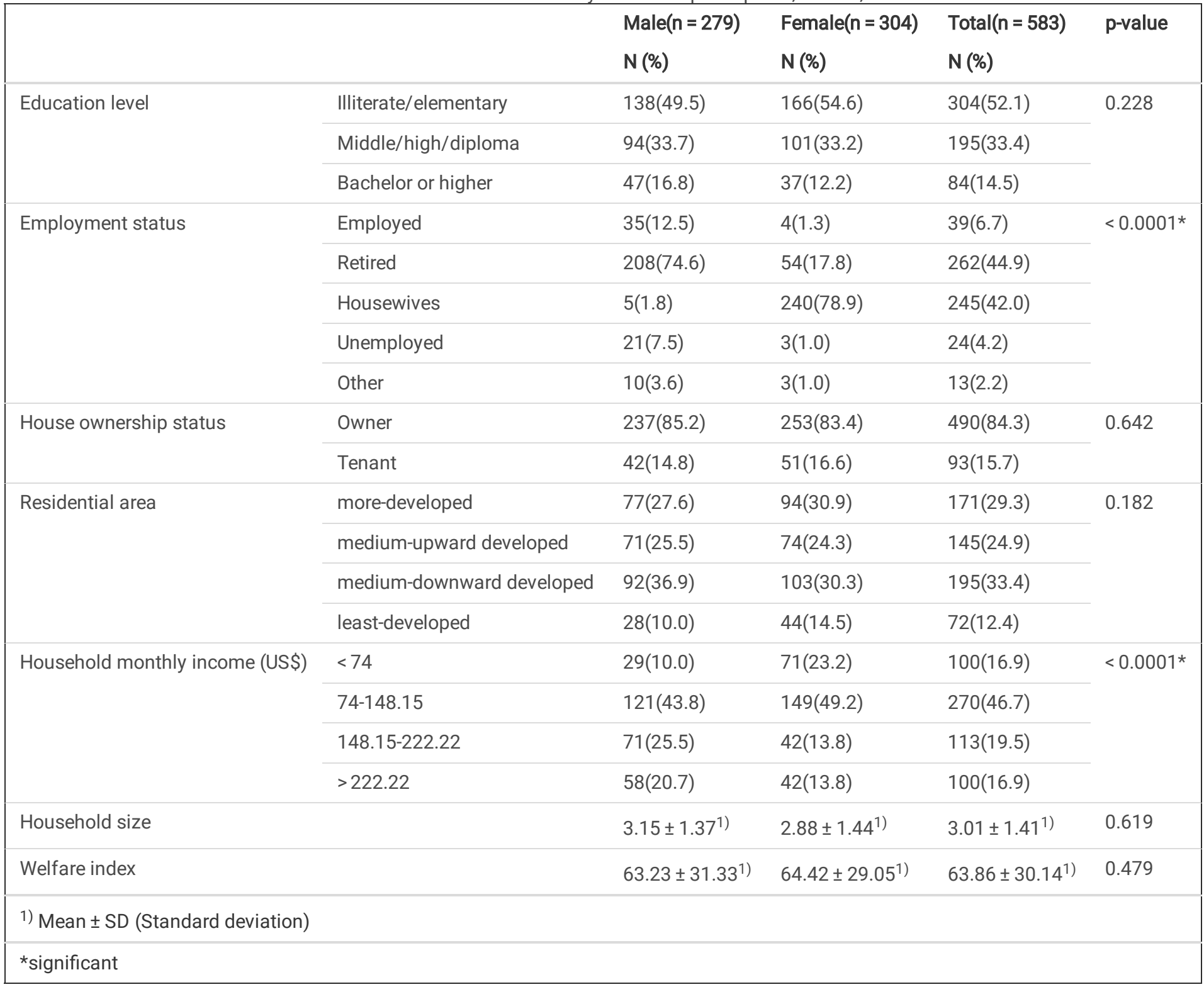

Table 3 displays food security status and DDS of the participants. After exclusion of misreporting, dietary diversity score was calculated for 511 participants. More than half of the participants were food secure (56.9\%). Only $17.4 \%$ of participants reported food insecurity (FI) with hunger in the past 12 months. About three quarters of the participants had high dietary diversity score (74.6\%). There was not any significant differences between men and women in terms of FI and DDS. 
Table 3

Food security status and dietary diversity score (DDS) by sex of the elderly participants, Tehran, Iran

\begin{tabular}{|c|c|c|c|c|c|}
\hline \multirow[t]{2}{*}{ Variable } & & Male $(n=279)$ & Female $(n=304)$ & Total $(n=583)$ & p-value \\
\hline & & $\mathbf{N}(\%)$ & $\mathbf{N}(\%)$ & $\mathbf{N}(\%)$ & \\
\hline \multirow[t]{4}{*}{ Food security status } & Food secure & 172(61.9) & $161(52.8)$ & $332(56.9)$ & \multirow[t]{4}{*}{0.122} \\
\hline & Food insecure without hunger & $67(23.8)$ & $83(27.6)$ & $150(25.7)$ & \\
\hline & Food insecure with moderate hunger & $32(11.0)$ & $51(16.3)$ & $83(14.2)$ & \\
\hline & Food insecure with severe hunger & $9(3.3)$ & $9(3.3)$ & $18(3.2)$ & \\
\hline \multirow[t]{2}{*}{$\left.\mathrm{DDS}^{1}\right)$} & $\operatorname{Low}(\leq 3)$ & $56(23.4)$ & $74(27.2)$ & $130(25.4)$ & \multirow[t]{2}{*}{0.328} \\
\hline & High (> 3) & 183(76.6) & 198(72.8) & $381(74.6)$ & \\
\hline
\end{tabular}

Based on Table 4, after adjusting for confounders in 2 models, the results of multinomial logistic regression indicated that being younger decreased the chance of food insecurity $(\mathrm{FI})$ with moderate hunger $(\mathrm{OR}=0.427,95 \% \mathrm{Cl}=0.206-0.886$ for adjusted model with sex; $\mathrm{OR}=0.317$, $95 \% \mathrm{Cl}=0.134-0.749$ for adjusted model with other cofounders) compared to those aged 75 to 80 years. In addition, being Fars decreased the possibility of being food insecure $(\mathrm{OR}=0.549,95 \% \mathrm{Cl}=0.324-931$ for Fl without hunger; $\mathrm{OR}=0.146,95 \% \mathrm{Cl}=0.051-0.424$ for $\mathrm{FI}$ with severe hunger) in comparison with other ethnicities. The number of children not living with the elderly were associated with higher chance of $\mathrm{FI}$ without hunger and $\mathrm{FI}$ with moderate hunger in unadjusted model $(\mathrm{OR}=1.171,95 \% \mathrm{Cl}=1.051-1.304)$ and $(\mathrm{OR}=1.224,95 \% \mathrm{Cl}=1.072-1.396)$, respectively which diminished in the adjusted model. Sex, marital status, living arrangement and the number of children living with the elderly were not significantly associated with food insecurity. 
Table 4

Unadjusted and adjusted association between demographic characteristics and food security status ${ }^{4}$ among the elderly living in Tehran, Iran $(n=583)$

\begin{tabular}{|c|c|c|c|c|c|c|c|c|c|}
\hline \multirow{2}{*}{$\begin{array}{l}\text { FS } \\
(n= \\
330)\end{array}$} & \multicolumn{3}{|c|}{$\begin{array}{l}\text { FI without hunger } \\
(n=148)\end{array}$} & \multicolumn{3}{|c|}{$\begin{array}{l}\text { FI with moderate hunger } \\
(n=81)\end{array}$} & \multicolumn{3}{|c|}{$\begin{array}{l}\text { FI with severe hunger } \\
(n=24)\end{array}$} \\
\hline & $\begin{array}{l}\text { OR } \\
(95 \% \mathrm{Cl})^{1} \\
\text { unadjusted }\end{array}$ & $\begin{array}{l}\text { OR } \\
(95 \% \mathrm{Cl}) \\
\text { adjusted }^{2}\end{array}$ & $\begin{array}{l}\text { OR } \\
(95 \% \mathrm{Cl}) \\
\text { adjusted }^{3}\end{array}$ & $\begin{array}{l}\text { OR } \\
(95 \% \mathrm{Cl})^{1} \\
\text { unadjusted }\end{array}$ & $\begin{array}{l}\text { OR } \\
(95 \% \mathrm{Cl}) \\
\text { adjusted }^{2}\end{array}$ & $\begin{array}{l}\text { OR } \\
(95 \% \mathrm{Cl}) \\
\text { adjusted }^{3}\end{array}$ & $\begin{array}{l}\text { OR } \\
(95 \% \mathrm{Cl})^{1} \\
\text { unadjusted }\end{array}$ & $\begin{array}{l}\text { OR } \\
(95 \% \mathrm{Cl}) \\
\text { adjusted }^{2}\end{array}$ & $\begin{array}{l}\text { OR } \\
(95 \% \mathrm{Cl}) \\
\text { adjusted }^{3}\end{array}$ \\
\hline \multicolumn{10}{|c|}{ Age (years) } \\
\hline $60-65$ & $\begin{array}{l}0.572 \\
(0.324- \\
1.010)\end{array}$ & $\begin{array}{l}0.618 \\
(0.342- \\
1.115)\end{array}$ & $\begin{array}{l}0.676 \\
(0.346-1.323)\end{array}$ & $\begin{array}{l}0.497 \\
(0.248- \\
0.998)^{\star}\end{array}$ & $\begin{array}{l}0.427 \\
(0.206- \\
0.886) \star\end{array}$ & $\begin{array}{l}0.317 \\
(0.134- \\
0.749) \star\end{array}$ & $\begin{array}{l}0.870 \\
(0.216- \\
3.506)\end{array}$ & $\begin{array}{l}0.908 \\
(0.216- \\
3.819)\end{array}$ & $\begin{array}{l}1.237 \\
(0.252- \\
6.079)\end{array}$ \\
\hline $66-75$ & $\begin{array}{l}0.663 \\
(0.387- \\
1.135)\end{array}$ & $\begin{array}{l}0.696 \\
(0.403- \\
1.203)\end{array}$ & $\begin{array}{l}0.675 \\
(0.374-1.219)\end{array}$ & $\begin{array}{l}0.609 \\
(0.318- \\
1.164)\end{array}$ & $\begin{array}{l}0.551 \\
(0.284- \\
1.070)\end{array}$ & $\begin{array}{l}0.417 \\
(0.199- \\
0.877)^{\star}\end{array}$ & $\begin{array}{l}1.153 \\
(0.312- \\
4.258)\end{array}$ & $\begin{array}{l}1.185 \\
(0.315- \\
4.456)\end{array}$ & $\begin{array}{l}1.477 \\
(0.364- \\
5.995)\end{array}$ \\
\hline $76-80$ & 1.00 Ref. & 1.00 Ref. & 1.00 Ref. & 1.00 Ref. & 1.00 Ref. & 1.00 Ref. & 1.00 Ref. & 1.00 Ref. & 1.00 Ref. \\
\hline \multicolumn{10}{|l|}{ Sex } \\
\hline Female & $\begin{array}{l}0.753 \\
(0.510- \\
1.113)\end{array}$ & $\begin{array}{l}0.897 \\
(0.368- \\
2.184)\end{array}$ & $\begin{array}{l}0.653 \\
(0.347-1.231)\end{array}$ & $\begin{array}{l}1.249 \\
(0.762- \\
2.047)\end{array}$ & $\begin{array}{l}1.467 \\
(0.873- \\
2.464)\end{array}$ & $\begin{array}{l}1.438 \\
(0.644- \\
3.212)\end{array}$ & $\begin{array}{l}0.877 \\
(0.370- \\
2.078)\end{array}$ & $\begin{array}{l}0.821 \\
(0.547- \\
1.232)\end{array}$ & $\begin{array}{l}1.367 \\
(0.297- \\
6.301)\end{array}$ \\
\hline male & 1.00 Ref. & 1.00 Ref. & 1.00 Ref. & 1.00 Ref. & 1.00 Ref. & 1.00 Ref. & 1.00 Ref. & 1.00 Ref. & 1.00 Ref. \\
\hline \multicolumn{10}{|c|}{ Marital status } \\
\hline single & $\begin{array}{l}1.178 \\
(0.727- \\
1.909)\end{array}$ & $\begin{array}{l}1.353 \\
(0.799- \\
2.292)\end{array}$ & $\begin{array}{l}1.274 \\
(0.650-2.500)\end{array}$ & $\begin{array}{l}1.542 \\
(0.874- \\
2.723)\end{array}$ & $\begin{array}{l}1.365 \\
(0.735- \\
2.535)\end{array}$ & $\begin{array}{l}1.607 \\
(0.744- \\
3.471)\end{array}$ & $\begin{array}{l}0.963 \\
(0.315- \\
2.944)\end{array}$ & $\begin{array}{l}0.998 \\
(0.999- \\
2.301)\end{array}$ & $\begin{array}{l}1.053 \\
(0.245- \\
4.535)\end{array}$ \\
\hline married & 1.00 Ref. & 1.00 Ref. & 1.00 Ref. & 1.00 Ref. & 1.00 Ref. & 1.00 Ref. & 1.00 Ref. & 1.00 Ref. & 1.00 Ref. \\
\hline \multicolumn{10}{|l|}{ Ethnicity } \\
\hline Fars & $\begin{array}{l}0.512 \\
(0.311- \\
0.841)^{\star}\end{array}$ & $\begin{array}{l}0.517 \\
(0.314- \\
0.853)^{\star}\end{array}$ & $\begin{array}{l}0.549 \\
(0.324- \\
0.931)^{\star}\end{array}$ & $\begin{array}{l}0.636 \\
(0.341-1.186)\end{array}$ & $\begin{array}{l}0.649 \\
(0.346- \\
1.217)\end{array}$ & $\begin{array}{l}0.648 \\
(0.330- \\
1.272)\end{array}$ & $\begin{array}{l}0.129 \\
(0.047- \\
0.353)^{\star}\end{array}$ & $\begin{array}{l}0.130 \\
(0.047- \\
0.358) \star\end{array}$ & $\begin{array}{l}0.146 \\
(0.051- \\
0.424)^{\star}\end{array}$ \\
\hline Azeri & $\begin{array}{l}1.215 \\
(0.687- \\
2.148)\end{array}$ & $\begin{array}{l}1.200 \\
(0.674- \\
2.134)\end{array}$ & $\begin{array}{l}0.824 \\
(0.443-1.532)\end{array}$ & $\begin{array}{l}1.186 \\
(0.575- \\
2.449)\end{array}$ & $\begin{array}{l}1.265 \\
(0.608- \\
2.634)\end{array}$ & $\begin{array}{l}0.802 \\
(0.363- \\
1.770)\end{array}$ & $\begin{array}{l}0.235 \\
(0.064- \\
0.866)^{\star}\end{array}$ & $\begin{array}{l}0.228 \\
(0.061- \\
0.850)^{\star}\end{array}$ & $\begin{array}{l}0.164 \\
(0.041- \\
0.654) \star\end{array}$ \\
\hline Other & 1.00 Ref. & 1.00 Ref. & 1.00 Ref. & 1.00 Ref. & 1.00 Ref. & 1.00 Ref. & 1.00 Ref. & 1.00 Ref. & 1.00 Ref. \\
\hline
\end{tabular}

\begin{tabular}{|l|}
\hline Ref., reference category. \\
\hline${ }^{1}$ Calculated by multinomial logistic regression. $95 \% \mathrm{Cl}$ : Confidence Interval of the 95\%.OR: odds radio. \\
\hline 2 Adjusted for age and sex. \\
\hline${ }^{3}$ Adjusted for age, sex, household income per month, education level, employment status, living arrangement, residential area. \\
\hline${ }^{4}$ The reference group for food security status is food secure. \\
\hline *significant, all statistical significance was tested at $\mathrm{P}<0.05$ level. \\
\hline
\end{tabular}




\begin{tabular}{|c|c|c|c|c|c|c|c|c|c|}
\hline \multirow{3}{*}{$\begin{array}{l}\quad \begin{array}{l}\text { FS } \\
\quad(n= \\
\quad 330)\end{array} \\
\text { With } \\
\text { family }\end{array}$} & \multicolumn{3}{|c|}{$\begin{array}{l}\text { FI without hunger } \\
(n=148)\end{array}$} & \multicolumn{3}{|c|}{$\begin{array}{l}\text { FI with moderate hunger } \\
(n=81)\end{array}$} & \multicolumn{3}{|c|}{$\begin{array}{l}\text { Fl with severe hunger } \\
(n=24)\end{array}$} \\
\hline & 0.821 & 0.775 & 0.900 & 0.866 & 1.025 & 1.031 & 1.237 & 1.247 & 1.469 \\
\hline & $\begin{array}{l}(0.454- \\
1.482)\end{array}$ & $\begin{array}{l}(0.420- \\
1.431)\end{array}$ & $(0.462-1.751)$ & $\begin{array}{l}(0.411- \\
1.825)\end{array}$ & $\begin{array}{l}(0.474- \\
2.220)\end{array}$ & $\begin{array}{l}(0.451- \\
2.357)\end{array}$ & $\begin{array}{l}(0.278- \\
5.508)\end{array}$ & $\begin{array}{l}(0.271- \\
5.744)\end{array}$ & $\begin{array}{l}(0.308- \\
7.017)\end{array}$ \\
\hline $\begin{array}{l}\text { Living } \\
\text { alone }\end{array}$ & 1.00 Ref. & 1.00 Ref. & 1.00 Ref. & 1.00 Ref. & 1.00 Ref. & 1.00 Ref. & 1.00 Ref. & 1.00 Ref. & 1.00 Ref. \\
\hline \multicolumn{10}{|c|}{ Relationship to the head of the household } \\
\hline \multirow[t]{2}{*}{ Spouse } & 0.710 & 0.812 & 0.878 & 0.726 & 0.525 & 0.406 & 0.675 & 0.619 & 0.516 \\
\hline & $\begin{array}{l}(0.464- \\
1.085)\end{array}$ & $\begin{array}{l}(0.458- \\
1.429)\end{array}$ & $(0.448-1.721)$ & $\begin{array}{l}(0.426- \\
1.238)\end{array}$ & $\begin{array}{l}(0.273- \\
1.009)\end{array}$ & $\begin{array}{l}(0.188- \\
0.876)^{\star}\end{array}$ & $\begin{array}{l}(0.257- \\
0.771)\end{array}$ & $\begin{array}{l}(0.183- \\
2.093)\end{array}$ & $\begin{array}{l}(0.130- \\
2.048)\end{array}$ \\
\hline Head & 1.00 Ref. & 1.00 Ref. & 1.00 Ref. & 1.00 Ref. & 1.00 Ref. & 1.00 Ref. & 1.00 Ref. & 1.00 Ref. & 1.00 Ref. \\
\hline Number & 1.083 & 1.101 & 1.092 & 1.054 & 1.115 & 1.100 & 1.004 & 1.011 & 0.977 \\
\hline $\begin{array}{l}\text { children } \\
\text { living } \\
\text { with the } \\
\text { elderly }\end{array}$ & $\begin{array}{l}(0.901- \\
1.301)\end{array}$ & $\begin{array}{l}(0.913- \\
1.328)\end{array}$ & $(0.894-1.333)$ & $\begin{array}{l}(0.837- \\
1.329)\end{array}$ & $\begin{array}{l}(0.882- \\
1.410)\end{array}$ & $\begin{array}{l}(0.855- \\
1.417)\end{array}$ & $\begin{array}{l}(0.661- \\
1.526)\end{array}$ & $\begin{array}{l}(0.660- \\
1.550)\end{array}$ & $\begin{array}{l}(0.630- \\
1.313)\end{array}$ \\
\hline Number & 1.171 & 1.165 & 1.049 & 1.224 & 1.200 & 1.054 & 1.126 & 1.120 & 0.966 \\
\hline $\begin{array}{l}\text { children } \\
\text { not } \\
\text { living } \\
\text { with the } \\
\text { elderly }\end{array}$ & $\begin{array}{l}(1.051- \\
1.304)^{\star}\end{array}$ & $\begin{array}{l}(1.042- \\
1.302)^{\star}\end{array}$ & $(0.923-1.193)$ & $\begin{array}{l}(1.072- \\
1.396)^{\star}\end{array}$ & $\begin{array}{l}(1.047- \\
1.375)^{\star}\end{array}$ & $\begin{array}{l}(0.897- \\
1.238)\end{array}$ & $\begin{array}{l}(0.890- \\
1.426)\end{array}$ & $\begin{array}{l}(0.879- \\
1.428)\end{array}$ & $\begin{array}{l}(0.738- \\
1.264)\end{array}$ \\
\hline \multicolumn{10}{|c|}{ Ref., reference category. } \\
\hline \multicolumn{10}{|c|}{${ }^{1}$ Calculated by multinomial logistic regression. $95 \% \mathrm{Cl}$ : Confidence Interval of the $95 \%$. R: odds radio. } \\
\hline \multicolumn{10}{|c|}{${ }^{2}$ Adjusted for age and sex. } \\
\hline \multicolumn{10}{|c|}{${ }^{3}$ Adjusted for age, sex, household income per month, education level, employment status, living arrangement, residential area. } \\
\hline \multicolumn{10}{|c|}{${ }^{4}$ The reference group for food security status is food secure. } \\
\hline
\end{tabular}

As shown in Table 5, earning less than 74 US $\$$ per month increased the risk of $\mathrm{Fl}$ without hunger $(\mathrm{OR}=4.240,95 \% \mathrm{Cl}=1.997-9.001)$ and $\mathrm{FI}$ with moderate hunger $(\mathrm{OR}=3.163,95 \% \mathrm{Cl}=1.296-7.716)$ in comparison with those with monthly income of higher than $222.22 \mathrm{US} \$$, respectively. In addition, those who lived in most-developed districts were at lower risk of food insecurity compared to other districts $(\mathrm{OR}=0.426,95 \% \mathrm{Cl}=$ $0.216-839$ for $\mathrm{Fl}$ without hunger; $\mathrm{OR}=0.397,95 \% \mathrm{Cl}=0.162-0.971 \mathrm{for} \mathrm{FI}$ with moderate hunger). Being retired was associated with a decreased risk of $\mathrm{Fl}$ without hunger and $\mathrm{FI}$ with moderate hunger after adjusting for age and $\operatorname{sex}(\mathrm{OR}=0.567,95 \% \mathrm{Cl}=0.325-0.989)$ and $(\mathrm{OR}=0.569$, $95 \% \mathrm{Cl}=0.337-0.960)$, respectively. Multinomial Logistic regression models suggested that after adjusting for age and sex, being illiterate increased the possibility of different levels of $\mathrm{FI}(\mathrm{OR}=3.107,95 \% \mathrm{Cl}=1.672-5.775),(\mathrm{OR}=2.736,95 \% \mathrm{Cl}=1.261-5.935)$ and $(\mathrm{OR}=6.193,95 \% \mathrm{Cl}$ $=1.368-28.039)$, respectively compared to those with high school diploma and higher degrees. For those with elementary to middle school education were significantly at higher risk of $\mathrm{FI}$ without hunger $(\mathrm{OR}=1.821,95 \% \mathrm{Cl}=1.150-2.883)$ and sever $\mathrm{FI}(\mathrm{OR}=3.813,95 \% \mathrm{Cl}=1.073-$ 13.546) in comparison with bachelor or higher education. House ownership status, household size and welfare index were not associated with $\mathrm{Fl}$. 
Table 5

Unadjusted and adjusted association between socio-economic characteristics and food security ${ }^{4}$ status among the elderly living in Tehran, Iran $(n=583)$

\begin{tabular}{|c|c|c|c|c|c|c|c|c|c|}
\hline \multirow{2}{*}{$\begin{array}{l}\text { FS } \\
(n=330) \\
\text { 1.00 Ref. }\end{array}$} & \multicolumn{3}{|c|}{$\begin{array}{l}\text { FI without hunger } \\
(n=148)\end{array}$} & \multicolumn{3}{|c|}{$\begin{array}{l}\text { FI with moderate hunger } \\
(n=81)\end{array}$} & \multicolumn{3}{|c|}{$\begin{array}{l}\text { FI with severe hunger } \\
(n=24)\end{array}$} \\
\hline & $\begin{array}{l}\text { OR } \\
(95 \% \mathrm{Cl})^{1} \\
\text { unadjusted }\end{array}$ & $\begin{array}{l}\text { OR } \\
(95 \% \mathrm{Cl}) \\
\text { adjusted }^{2}\end{array}$ & $\begin{array}{l}\text { OR } \\
(95 \% \mathrm{Cl}) \\
\text { adjusted }^{3}\end{array}$ & $\begin{array}{l}\text { OR } \\
(95 \% \mathrm{Cl})^{1} \\
\text { unadjusted }\end{array}$ & $\begin{array}{l}\text { OR } \\
(95 \% \mathrm{Cl}) \\
\text { adjusted }^{2}\end{array}$ & $\begin{array}{l}\text { OR } \\
(95 \% \mathrm{Cl}) \\
\text { adjusted }^{3}\end{array}$ & $\begin{array}{l}\text { OR } \\
(95 \% \mathrm{Cl})^{1} \\
\text { unadjusted }\end{array}$ & $\begin{array}{l}\text { OR } \\
(95 \% \mathrm{Cl}) \\
\text { adjusted }^{2}\end{array}$ & $\begin{array}{l}\text { OR } \\
(95 \% \mathrm{Cl}) \\
\text { adjusted }^{3}\end{array}$ \\
\hline \multicolumn{10}{|l|}{ Education level } \\
\hline Illiterate & $\begin{array}{l}3.224 \\
(1.770- \\
5.872)^{\star}\end{array}$ & $\begin{array}{l}3.107 \\
(1.672- \\
5.775)^{\star}\end{array}$ & $\begin{array}{l}1.662 \\
(0.783- \\
3.530)\end{array}$ & $\begin{array}{l}3.155 \\
(1.495- \\
6.659)^{\star}\end{array}$ & $\begin{array}{l}2.736 \\
(1.261- \\
5.935)^{\star}\end{array}$ & $\begin{array}{l}1.156 \\
(0.446- \\
2.997)\end{array}$ & $\begin{array}{l}5.877 \\
(0.343- \\
27.715)^{\star}\end{array}$ & $\begin{array}{l}6.193 \\
(1.368- \\
28.039)^{\star}\end{array}$ & $\begin{array}{l}3.632 \\
(0.627- \\
21.038)\end{array}$ \\
\hline $\begin{array}{l}\text { Elementary to } \\
\text { middle school }\end{array}$ & $\begin{array}{l}1.821 \\
(1.150- \\
2.883)^{\star}\end{array}$ & $\begin{array}{l}1.805 \\
(1.131- \\
2.880)^{\star}\end{array}$ & $\begin{array}{l}1.175 \\
(0.676- \\
2.042)\end{array}$ & $\begin{array}{l}1.892 \\
(1.055- \\
3.395)^{\star}\end{array}$ & $\begin{array}{l}1.795 \\
(0.992- \\
3.248)\end{array}$ & $\begin{array}{l}0.915 \\
(0.452- \\
1.852)\end{array}$ & $\begin{array}{l}3.813 \\
(1.073- \\
13.546)^{*}\end{array}$ & $\begin{array}{l}3.867 \\
(1.076- \\
13.903)^{*}\end{array}$ & $\begin{array}{l}2.905 \\
(0.675- \\
12.504)\end{array}$ \\
\hline $\begin{array}{l}\text { Diploma or } \\
\text { higher }\end{array}$ & 1.00 Ref. & 1.00 Ref. & 1.00 Ref. & 1.00 Ref. & 1.00 Ref. & 1.00 Ref. & 1.00 Ref. & 1.00 Ref. & 1.00 Ref. \\
\hline \multicolumn{10}{|c|}{ Employment status } \\
\hline Employed & $\begin{array}{l}0.936 \\
(0.408- \\
2.145)\end{array}$ & $\begin{array}{l}0.640 \\
(0.246- \\
1.666)\end{array}$ & $\begin{array}{l}0.778 \\
(0.287- \\
2.113)\end{array}$ & $\begin{array}{l}1.123 \\
(0.449- \\
2.809)\end{array}$ & $\begin{array}{l}1.423 \\
(0.471- \\
4.303)\end{array}$ & $\begin{array}{l}1.633 \\
(0.517- \\
5.155)\end{array}$ & $\begin{array}{l}1.476 \\
(0.303- \\
7.204)\end{array}$ & $\begin{array}{l}1.384 \\
(0.208- \\
9.218)\end{array}$ & $\begin{array}{l}2.390 \\
(0.306- \\
18.658)\end{array}$ \\
\hline Retired & $\begin{array}{l}0.887 \\
(0.593- \\
1.326)\end{array}$ & $\begin{array}{l}0.567 \\
(0.325- \\
0.989) *\end{array}$ & $\begin{array}{l}0.775 \\
(0.406- \\
1.479)\end{array}$ & $\begin{array}{l}0.569 \\
(0.337- \\
0.960)^{\star}\end{array}$ & $\begin{array}{l}0.539 \\
(0.266- \\
1.092)\end{array}$ & $\begin{array}{l}0.611 \\
(0.272- \\
1.374)\end{array}$ & $\begin{array}{l}0.969 \\
(0.392- \\
2.392)\end{array}$ & $\begin{array}{l}0.862 \\
(0.256- \\
2.898)\end{array}$ & $\begin{array}{l}1.981 \\
(0.417- \\
9.401)\end{array}$ \\
\hline Unemployed & 1.00 Ref. & 1.00 Ref. & 1.00 Ref. & 1.00 Ref. & 1.00 Ref. & 1.00 Ref. & 1.00 Ref. & 1.00 Ref. & 1.00 Ref. \\
\hline \multicolumn{10}{|c|}{ House ownership status } \\
\hline Tenant & $\begin{array}{l}1.347 \\
(0.790- \\
2.296)\end{array}$ & $\begin{array}{l}1.374 \\
(0.804- \\
2.348)\end{array}$ & $\begin{array}{l}1.344 \\
(0.755- \\
2.393)\end{array}$ & $\begin{array}{l}0.924 \\
(0.444- \\
1.923)\end{array}$ & $\begin{array}{l}0.949 \\
(0.454- \\
1.983)\end{array}$ & $\begin{array}{l}0.975 \\
(0.449- \\
2.119)\end{array}$ & $\begin{array}{l}1.021 \\
(0.290- \\
3.591)\end{array}$ & $\begin{array}{l}1.017 \\
(0.289- \\
3.581)\end{array}$ & $\begin{array}{l}0.942 \\
(0.255- \\
3.482)\end{array}$ \\
\hline Owner & 1.00 Ref. & 1.00 Ref. & 1.00 Ref. & 1.00 Ref. & 1.00 Ref. & 1.00 Ref. & 1.00 Ref. & 1.00 Ref. & 1.00 Ref. \\
\hline \multicolumn{10}{|c|}{ Household income per month (US\$) } \\
\hline$<74$ & $\begin{array}{l}3.827 \\
(1.839- \\
7.965)^{\star}\end{array}$ & $\begin{array}{l}4.240 \\
(1.997- \\
9.001)^{\star}\end{array}$ & $\begin{array}{l}2.049 \\
(0.883- \\
4.751)\end{array}$ & $\begin{array}{l}3.163 \\
(1.296- \\
7.716)^{\star}\end{array}$ & $\begin{array}{l}2.744 \\
(1.100- \\
6.846)^{\star}\end{array}$ & $\begin{array}{l}1.131 \\
(0.397- \\
3.224)\end{array}$ & $\begin{array}{l}3.349 \\
(0.588- \\
19.059)\end{array}$ & $\begin{array}{l}3.858 \\
(0.658- \\
22.622)\end{array}$ & $\begin{array}{l}1.955 \\
(0.289- \\
13.222)\end{array}$ \\
\hline $74-148.15$ & $\begin{array}{l}2.571 \\
(1.358- \\
4.870)^{\star}\end{array}$ & $\begin{array}{l}2.637 \\
(1.379- \\
5.043)^{\star}\end{array}$ & $\begin{array}{l}1.737 \\
(0.871- \\
3.467)\end{array}$ & $\begin{array}{l}1.944 \\
(0.887- \\
4.264)\end{array}$ & $\begin{array}{l}1.738 \\
(0.784- \\
3.855)\end{array}$ & $\begin{array}{l}1.052 \\
(0.441- \\
2.512)\end{array}$ & $\begin{array}{l}3.750 \\
(0.835- \\
16.845)\end{array}$ & $\begin{array}{l}4.051 \\
(0.889- \\
18.466)\end{array}$ & $\begin{array}{l}2.562 \\
(0.525- \\
12.497)\end{array}$ \\
\hline
\end{tabular}

Ref., reference category.

${ }^{1}$ Calculated by multinomial logistic regression. $95 \% \mathrm{Cl}$ : Confidence Interval of the $95 \% .0 \mathrm{R}$ : odds radio.

${ }^{2}$ Adjusted for age and sex.

${ }^{3}$ Adjusted for age, sex, household income per month, education level, employment status, living arrangement, residential area.

4 The reference group for food security status is food secure.

*significant, all statistical significance was tested at $\mathrm{P}<0.05$ level. 


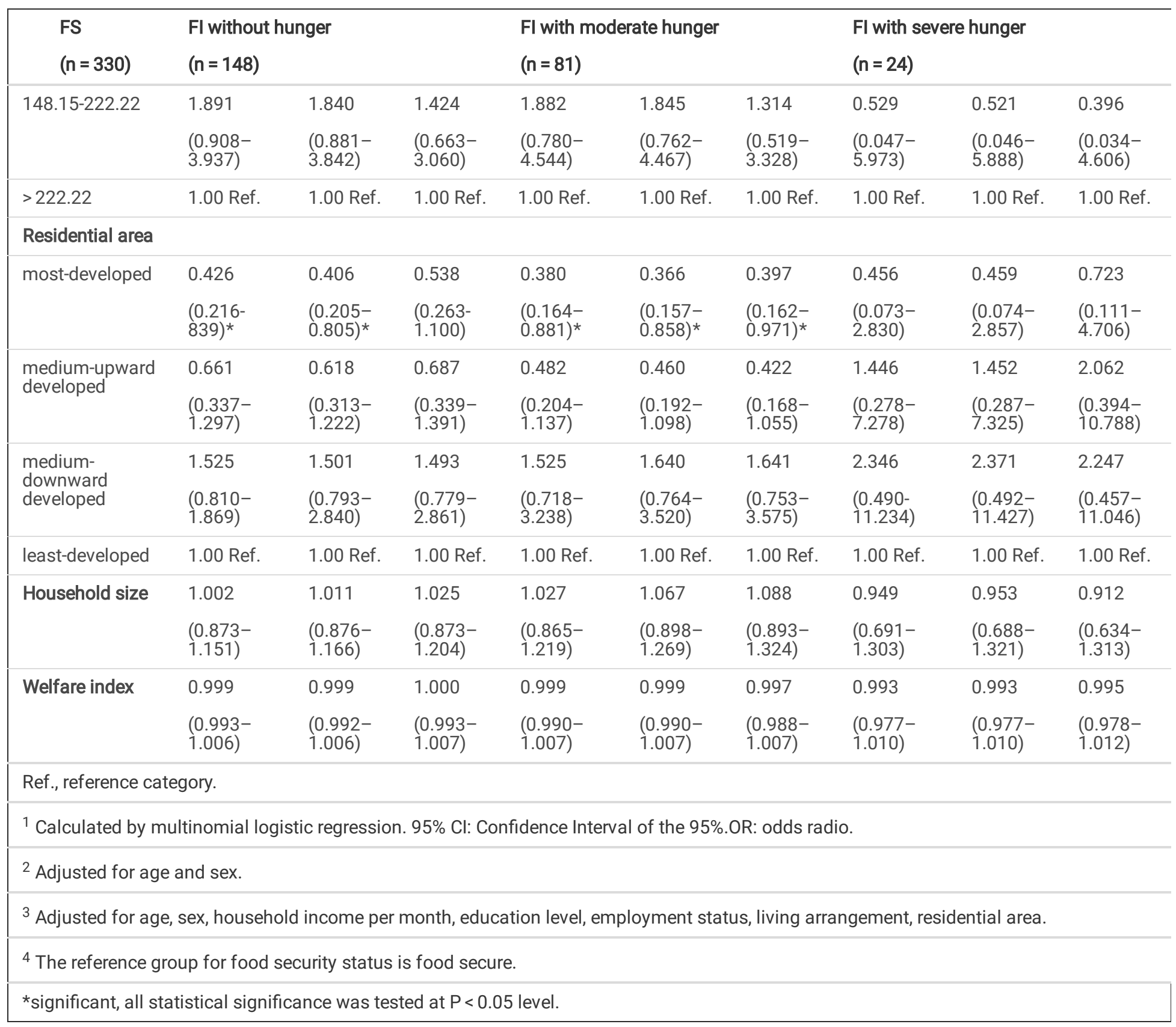

Table 6, presents the results from unadjusted and adjusted binary regression analyses of the associations between dietary diversity score (DDS) and food security status. The elderly who were food insecure with severe hunger had less odds to be in high DDS group; however, the association was not significant. 
Table 6

Unadjusted and adjusted association between dietary diversity score (DDS) with food security status ${ }^{4}$ among the elderly living in Tehran, Iran $(n=511)$

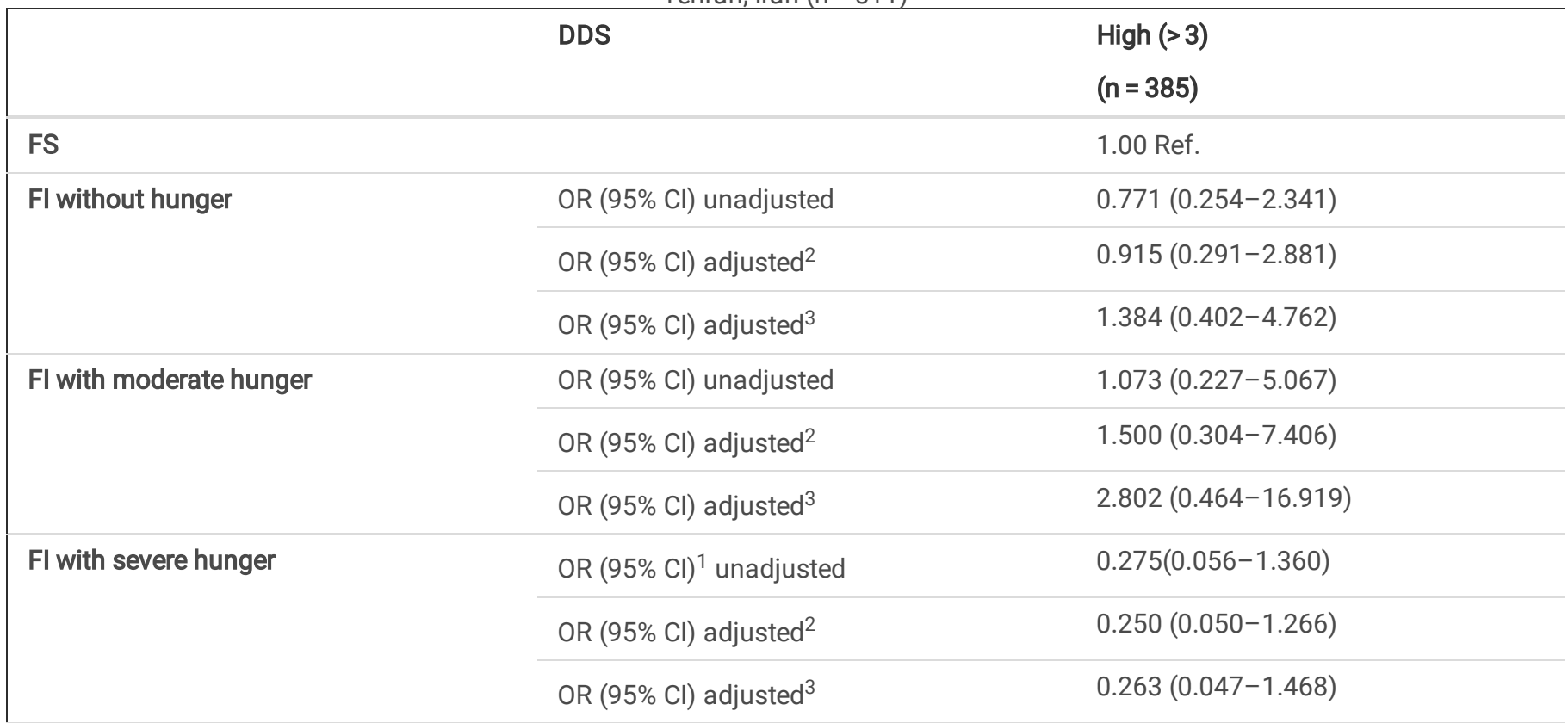

Ref., reference category.

${ }^{1}$ Calculated by binary logistic regression. $95 \% \mathrm{Cl}$ : Confidence Interval of the $95 \%$. OR: odds radio.

${ }^{2}$ Adjusted for age and sex.

${ }^{3}$ Adjusted for age, sex, household income per month, education level, employment status, living arrangement, and residential area.

4 The reference group for food security status is food secure.

* significant, all statistical significance was tested at $\mathrm{P}<0.05$ level.

\section{Discussion And Conclusion}

The results of the present study showed a significant association between the level of household income and the severity of Fl among the urban free-living elderly, suggesting that income is the most important predictor of food security status in this age group. Other predictors of reduced risk of FI without hunger were being retired, being Fars (the dominant ethnic group in the country) and living in affluent districts of the city. The determinants associated with reduced risk of FI with moderate hunger were age (being younger), living in most-developed areas, not being the head of the household and fewer number of children not living with the elderly. The only determinant associated with reduced risk of FI with severe hunger was elderly s ethnicity (being Fars or Azeri). There was no association between FI and DDS, even after controlling for confounders.

The findings are consistent with other studies that have suggested a relationship between income and food insecurity [33, 38-39]. As, a direct relationship between income changes and changes in food sufficiency has been shown in a study in Michigan [33], this study documented that changes in income and employment status were related to changes in severity of food insecurity as measured by HFSSM. Elderlies who received lower income faced 4 times higher odds of being FI without hunger and 2 times higher odds of being FI with mild hunger compared with those who had sufficient income. These findings suggest that income has a crucial role in sustaining food security for elderly individuals. Effects of adequate income on food security were noted in existing literatures [18, 24-25, 30-31]. However, this study found that there was not significant relationship between lower income and FI with severe hunger. The voluminous number of positive responds on the HFSSM module has been used as outcome variable in other studies [32,34-36]. This study also showed that a change in individual food insecurity scale score is an important indicator of economic changes within households.

Another unique finding was that illiterate elderlies and those with less than secondary education faced 6 times and more than 3 times higher odds of being FI with severe hunger compared to those with Bachelor or higher education, respectively. In fact, the highest prevalence as well as the greatest severity of food insecurity was observed among illiterate elderlies. This factor could reflect an important limitation for illiterate 
elderlies to gain higher level job and poorer quality of current job. Because getting any or additional job and increasing work hours may not be possible for many elderlies, particularly those with low educational levels.

The findings suggest that being retired reduced the chance of food insecurity among the elderlies compared with those who were unemployed or their previous jobs did not provide retirement. The relationship between a gain in employment and reduction in severity of food insecurity have been observed in other studies, as well [30-32]. Retired seniors are probably better off financially compared to people who still have to work at this age.

Whereas previous studies showed that elderlies, with more children living with them were more vulnerable to food insecurity [24, 29], in the current study, elderlies who had children not living with them were at higher risk of food insecurity. Although household size and marital status was not related with food security in the present study, a recent study of food security among households explained that households with single women, larger household size, and low incomes were more likely to be food insecure [37]. Older people are poorer physically and functionally than younger ones, affecting their ability to cook, consume and absorb food. In addition, factors such as living alone or with fewer children, as a sign of being alone or lack of support can lead to increased chances of food insecurity in this age group [24, 47-48].

Respondents in the most-developed residential districts were significantly less likely to experience food insecurity even in the mild form. Although a cross-sectional study in Australia did not find significant association between food insecurity and residential area [24], most studies approve the fact that living in wealthy areas was negatively associated with FI risk [25, 41, 43].

The present results showed that the risk of food insecurity was lower if elderly were Fars and Azeri. A cross-sectional study which has done in Arak, central Iran, also supports this observation [30]. The relationship between being Azeri and reduction the risk of food insecurity has been observed in other studies in Iran [41]. A study has shown that the Azeri people have a good social economic status [41-42]. Higher food security of the Fars people was due to being a dominant ethnic group, as well as being Azeri who are likely to be immigrants with longer residence time and better job prospects and higher life expectancy compared to other ethnicities.

The present study showed that marital status and gender were not related to elderly's food security status, even after adjusting for cofounders. Other studies also found no association between marital status and gender with food security status in elderlies [24, 30].

Although the relationship between dietary diversity and food security status was not significant in the present study, several studies have found this inverse association to be significant $[43,45]$. A study in Iran has shown that the mean DDS of participants in the high food security group was significantly higher than the food insecure group [19]. A cross-sectional study in Taiwan showed that dietary diversity score was negatively associated with elderlies' food security status, especially with regard to meat group DDS [44]. The lack of a significant relationship between food security status and DDS in this study, may be due to the fact that FAO (2013) instructions for calculating the DDS is easy without considering the servings of food groups consumption.

In conclusion, this study shows that income, employment status and educational levels of elderly were the strongest predictors of food insecurity and its severity, highlighting the sensitivity of food-insecurity to socio-economic characteristics. These findings suggest that more household monthly income and better employment status of elderly would be associated with more food security. The results support the need for development of public policy aimed at improving the basic resources of food-insecure elderly to improve the severity of their experiences. Also, it calls for an instant need for development of a policy aimed at identifying and supporting food-insecure elderly in Tehran and other metropolitans in the country, as currently there is no public policy in place to tackle this problem. Development of assistance programs for lowincome and less educated elderly is recommended. This study provides support for improving availability of secure employment opportunities and facilitating the retirement process for the elderly.

The large sample of the present study provided an opportunity for investigating the association between socio-economic and demographic characteristics and severity of food insecurity. Also, it provided a useful snapshot of free living elders` FI status and its approximate determinants. The very high response rate (583 participants) is also a notable strength of our study. However, due to the cross-sectional design of the study, we were unable to assess any bias that were from non-responders. The cross-sectional design also prevents an analysis of temporal association and causality. Since nutritional outcomes could inversely affect food security determinants, reverse causality is possible. Prospective monitoring of food security and its determinants is required to clarify the direction of causation. Also, the measures used do not quantify all hypothetical determinants; this limits the study in the comprehensiveness of the analysis and possibility of missing cofounders. Educational levels, employment status and other factors may be a product of financial and nonfinancial limitations directly related to food security and nutrition, and therefore the estimates of this study cannot be explained as cause and effect. US-HFSSM is limited its representation of 'individual food security' because it screen the respondents' perception of whether they had enough food; HFSSM might only measure calorie, but not micronutrient.

\section{Abbreviations}




\section{Declarations}

\section{Ethics approval and consent to participate}

Ethical approval was obtained from the National Nutrition and Food Technology Research Institute, Shahid Beheshti University of Medical Sciences. (IR.SBMU.NNFTRI.REC.1397.052) Informed consent was obtained from the respondents (informed and written).

\section{Consent for publication}

Not applicable

\section{Availability of data and material}

Please contact author for data requests.

\section{Competing interests}

The authors declare that they have no competing interests.

\section{Funding}

This research is funded by National Nutrition and Food Technology Research Institute.

\section{Authors' contributions}

NO and AR designed the study; FP and PS conducted the study and entered data in statistical software; FP analyzed the data; FP wrote the paper; NO and AR contributed to interpretation of the data, reviewed and edited the manuscript; HE assisted in the study analyses. All authors read and approved the final manuscript.

\section{Acknowledgements}

We gratefully acknowledge all of the participants in this study.

\section{References}

1. National Elderly Document: the aging population of Iran, challenge and opportunity for all. Ministry of Health and Medical Education. Tehran, Iran; 2018. http://snce.ir/wp-content/uploads/2018/07/national-elderly-document-in-2018.pdf.

2. Khoshbin S, Rahimi A, Nvayyan N. Iran's Population Prediction 2000-5050. Ministry of Health and Medical Education. Tehran; 2004.

3. Habibi A, Neekpoor S, Seyedolshohda M, Haghani H. Health promotion behaviours and Quality of life among elderly people: A crosssectional survey 2006. Journal of Ardabil University of Medical Sciences. 2008;8(1):29-36.

4. Beard JR, Officer AM, Cassels AK. The World Report on Ageing and Health. The Gerontologist. 2016;56Suppl2:S163-6. doi:10.1093/geront/gnw037.

5. Heidari M, Shahbazi S. Effect of self-care training program on quality of life of elders. Iran Journal of Nursing. 2012;25(75):1-8.

6. Campbell CC. Food insecurity: A nutritional outcome or a predictor variable. Journal of Nutrition. 1991;121(3):408. doi:10.1093/jn/121.3.408.

7. FAO's Agriculture and Development Economics Division (ESA) with support from the FAO Netherlands Partnership Programme (FNPP) and the EC-FAO Food Security Programme. Food security. Policy Br. Last modified June 2006. http://www.fao.org/fileadmin/templates/faoitaly/documents/pdf/pdf_Food_Security_Cocept_Note.pdf. Accessed September 27, 2018.

8. Wicks R, Trevena LJ, Quine S. Experiences of Food Insecurity among Urban Soup Kitchen Consumers: Insights for Improving Nutrition and Well-Being. J Am Diet Assoc. 2006;106(6):921-4. doi:10.1016/j.jada.2006.03.006. 
9. Lee JS, Frongillo EA. Nutritional and health consequences are associated with food insecurity among US elderly persons. J Nutr. 2001;131:1503-9. doi:10.1093/jn/131.5.1503.

10. Food Prices, Nutrition, and the Millennium Development Goals. The International Bank for Reconstruction and Development. Washington, DC: The World Bank; 2012.

11. Dastgiri S, Soltani M, Ostad rahimi A, Tootoonchi H. Factors affecting food insecurity: A study in Tabriz. J Ardabil Univ Med Sci. 2006;6:233-9.

12. Barrett CB. Foodsecurityandfoodassistanceprograms. 1st ed. in: Gardner BL \& Rausser GC (ed.). Handbook of Agricultural Economics; 2002.

13. Azadbakht L, Esmaillzadeh A. Dietary diversity score is related to obesity and abdominal adiposity among Iranian female youth. Public Health Nutr. 2011;14(1):62 - 9. doi: 10.1017/S1368980010000522.

14. Fathi H. World agriculture: towards 2015-2030: an FAO perspective. Ministry of Jihad Agriculture. Tehran, Iran; 2003.

15. Weimer JP. Many elderly at nutritional risk. Economic Research Service. Washington, DC; 1997.

16. Hoddinatt, J. Choosing Outcome Indicators of Household Food Security in Methods for Rural Development Projects. International Food Policy Research Institute (IFPRI). Washington, DC; 1999.

17. Thiele S, Weiss C. Consumer demand for food diversity: evidence for Germany. Food Policy. 2003;28(2):99-115. doi:10.1016/S03069192(02)00068-4.

18. Tavakoli S, Dorosty-motlagh AR, Hoshiar-Rad A, Eshraghian MR, Sotoudeh G, Azadbakht L, et al. Is dietary diversity a proxy measurement of nutrient adequacy in Iranian elderly women. Appetite. 2016;105:468-76. doi: 10.1016/j.appet.2016.06.011.

19. Ghomi M H, Mirmiran P, et al. The association of food security with dietary diversity in people over 40 years of age. Iranian Journal of Endocrinology and Metabolism. 2012;14(4):360-367.

20. Telikani Sh. Food security status and effector policies in urban and rural areas. [MS Thesis] Shiraz: Shiraz Uni; 2006.

21. Kim J, Lee Y, Kye S, Chung YS, Kim JH, Chon D, et al. Diet quality and osteosarcopenic obesity in community-dwelling adults 50 years and older. Maturitas. 2017;104:73-9. doi: 10.1016/j.maturitas.2017.08.007.

22. Rafiei M, Nord M, Sadeghizadeh A, Entezari MH. Assessing the internal validity of a household survey-based food security measure adapted for use in Iran. Nutrition Journal. 2009;8:28. doi: 10.1186/1475-2891-8-28.

23. Bickel G, Nord M, Price C, Hamilton W, Cook J. Guide to Measuring Household Food Security, revised 2000. US Department of Agriculture, Food and Nutrition Service. Alexandria VA; 2000. https://fns-prod.azureedge.net/sites/default/files/FSGuide.pdf. Accessed May 7, 2018.

24. Quine S, Morrell S. Food insecurity in community-dwelling older Australians. Public Health Nutrition. 2006;9(2):219-24. doi:10.1079/phn2005834.

25. Lee JS, Frongillo EA. Factors Associated With Food Insecurity Among U.S. Elderly Persons: Importance of Functional Impairments. The Journals of Gerontology Series B: Psychological Sciences and Social Sciences. 2001;56(2):94-9. doi:10.1093/geronb/56.2.s94.

26. Bowman SA, Gortmaker SL, Ebbeling CB, Pereira MA, Ludwig DS. Effects of Fast-Food Consumption on Energy Intake and Diet Quality among Children in a National Household Survey. Pediatrics. 2004;113(1):112. doi:10.1542/peds.113.1.112.

27. Ghafarpour M, Houshiar-Rad A, Kianfar H. Directory Home Scales, Coefficients and Percentage of Edible Food. Agriculture Sciences. Tehran, Iran; 1378.

28. Mirzaei J B, Ahmadi S, Lorestanie A. Spatial analysis of urban metropolitan areas occupancy levels from the perspective of urban economics. Journal of Urban Economics and Management. 2015;3(11):59-77.

29. Milani-Bonab A, Mozaffari-Khosravi H, Omidvar N. Assessing the Relationship between Food Insecurity \& Weight Status of the Elderly applying FACPS-FSSM Measurement Tool, adopted for Iranian elderly. Nutrition and Food Sciences Research. $2014 ; 1$ (SUPPL.(1)):42.

30. Fallah Tafti L, Mozafari Khosravi H, Najarzadeh A, Falahzadeh H. Food Security Status of Elders and Its Related Factors in Arak in 2012. TB. 2016;14(5):64-78.

31. Souza Bruna Fernanda do Nascimento Jacinto de, Marín-León Letícia. Food insecurity among the elderly: Cross-sectional study with estudo transversal com usuários de restaurante popular. Rev Nutr. 2013;26(6):679-91. doi:10.1590/S1415-52732013000600007.

32. Loopstra R, Tarasuk V. Severity of Household Food Insecurity Is Sensitive to Change in Household Income and Employment Status among Low-Income Families. J Nutr. 2013;143(8):1316-23. doi: 10.3945/jn.113.175414.

33. Heflin CM, Corcoran ME, Siefert KA. Work trajectories, income changes, and food insufficiency in a Michigan welfare population. Soc Serv Rev. 2007;81(1):3-25. doi:10.1086/511162.

34. Bhargava A, Jolliffe D, Howard LL. Socio-economic, behavioral and environmental factors predicted body weights and household food insecurity scores in the Early Childhood Longitudinal Study-Kindergarten. Br J Nutr. 2008;100:438-44. doi: 10.1017/S0007114508894366. 
35. Huang J, Guo B, Kim Y. Food insecurity and disability: Do economic resources matter. Social Science Research. 2010;39:111-24. doi:10.7936/K7GB23KN.

36. Guo G. Household assets and food security: evidence from the survey of program dynamics. J Fam Econ Issues. 2011;32:98-110. doi: 10.1007/s10834-010-9194-3.

37. Mclntyre L, Bartoo AC, Emery JH. When working is not enough: food insecurity in the Canadian labour force. Public Health Nutrition. 2012;17(1):49-57. doi: 10.1017/S1368980012004053.

38. Gundersen C, Ribar D. The dynamic determinants of food insufficiency. In: Andrews MS, Prell MA. Second food security measurement and research conference. Economic Research Service, U.S. Department of Agriculture. Food assistance and nutrition research report. Washington, DC; 2001.

39. Leete L, Bania N. The effect of income shocks on food insufficiency. Rev Econ Househ. 2010;8:505-26. doi:10.1007/s11150-009-9075-4.

40. Kennedy G, Ballard T, Dop MC. Guidelines for Measuring Household and Individual Dietary Diversity. Food and Agriculture Organization of the United Nations; 2011. http://www.fao.org/fileadmin/user_upload/wa_workshop/docs/FAO-guidelines-dietary-diversity2011.pdf. Accessed May 17, 2018.

41. 10.1080/13557858.2015.1061102

Rezazadeh A, Omidvar N, Eini-Zinab H, Ghazi-Tabatabaie M, etc. Food insecurity, socio-economic factors and weight status in two Iranian ethnic groups. Ethnicity \& health. 2016;21(3):233-250. doi: 10.1080/13557858.2015.1061102.

42. Rezazadeh A, Rashidkhani B, Omidvar N. Association of major dietary patterns with socioeconomic and lifestyle factors of adult women living in Tehran, Iran. Nutrition. 2010;26(3):337-341. doi: 10.1016/j.nut.2009.06.019.

43. McDonald CM, McLean J, Kroeun H, Talukder A, Lynd LD, Green TJ. Correlates of household food insecurity and low dietary diversity in rural Cambodia. Asia Pac J Clin Nutr. 2015;24(4):720-30. doi: 10.6133/apjcn.2015.24.4.14.

44. Lo YT, Chang YH, Lee MS, Wahlqvist ML. Dietary diversity and food expenditure as indicators of food security in older Taiwanese. Appetite. 2012;58(1):180-7. doi: 10.1016/j.appet.2011.09.023.

45. Tanhaei M, Zare A, Shirani Bidabadi F, Jolai R. Survey of Food Security Status Using Diversity Index: A Case Study of Rural Areas of Marvdasht, Fars Province. Village and Development. 1394;18(4):17-35.

46. Milani-Bonab A, Mozaffari-Khosravi H, Omidvar H. Assessment of Content Validity and Internal Consistency and Reliability of Direct Experience-Based Elderly Food Insecurity Measurement Tool for Iranian Elders. Tolooebehdasht. 2012;11(2):57-74.

47. Kim K, Kim MK, Shin Y, Lee SS. Factors related to household food insecurity in the Republic of Korea. Public Health Nutrition. 2011;14(6):1080-7. doi: 10.1017/S1368980010003733.

48. Wolfe WS, Olson CM, Kendall A, Frongillo EA. Understanding Food Insecurity in the Elderly: A Conceptual Framework. Journal of Nutrition Education. 1996;28(2):92-100. doi:10.1093/jn/133.9.2762. 